\title{
INVASIVE DUCTAL CARCINOMA IN A PATIENT WITH LI-FRAUMENI SYNDROME: A CASE REPORT
}

Gabriel Baêta Branquinho Reis', Hugo Francisco da Fonseca Neto', Alice Jardim Zaccariotti', Daniel Bispo de Sousa', Silvaleide Ataides Assunção', Thiago Martins de Abreu', Fernando Santos de Azevedo², Lanúscia Morais de Santana²

${ }^{1}$ Universidade Federal de Goiás - Goiânia (GO), Brazil.

${ }^{2}$ Hospital das Clínicas, Universidade Federal de Goiás - Goiânia (GO), Brazil.

Introduction/Objectives: Breast cancer is one of the most common malignancies among women, with $10 \%$ resulting from genetic predisposition. Li-Fraumeni syndrome is an autosomal dominant disease that predisposes to multiple primary tumors and is responsible for less than $0.1 \%$ of breast cancers, being considered in early-onset tumors. The aim of this report was to describe a fast evolution of three primary tumors in a young patient with Li-Fraumeni syndrome, including ductal breast carcinoma. Case Report: In 2017, a 27-year-old female patient was diagnosed with malignant cancer of the right breast, Luminal HER KI67 70\%, clinical stage IV (liver and lung), underwent first-line cancer treatment, maintaining endocrinotherapy and Double Block, with a positive genetic panel test for TP53 mutation, inferring SLF. In 2018, screening colonoscopy showed colon adenocarcinoma, pT53pN1, treated with total colectomy with ileal pouch, followed by suspension of endocrinotherapy and maintenance of Double Block and adjuvant FOLFOX. At the end of chemotherapy, endocrinotherapy was adopted again. Reassessment tests showed partial response in the liver, but the primary nodules were unchanged. Biopsy after thoracoscopy described lung adenocarcinoma, pT3pN2, submitted to adjuvant with Gemzar and Navelbine, followed by Double Block and interruption of endocrinotherapy. It evolved with the appearance of nodules in the right breast, suggestive of progression of breast disease, under treatment with Xeloda, Herceptin, and Perjeta, showing good clinical response. Discussion: Breast cancer in young people increases the possibility of heredity, thus raising the need for investigations of genetic syndromes. Although rare, the identification of FHL brings an important implication for the genetic counseling. Early diagnosis is the best form of management, enabling the preventive screening and intervention of multiple malignancies. Conclusion: Cases of breast cancer in young women should raise a suspected diagnosis of Li-Fraumeni syndrome, which can change the therapeutic and investigation of other cancers at an early stage.

Keywords: Li-Fraumeni Syndrome; Breast Cancer. 